\title{
Spectral Features: how to reduce them
}

\author{
Hedser van Brug*a, Daniel ten Bloemendal ${ }^{\mathrm{a}}$, Bryan de Goeij ${ }^{\mathrm{a}}$, Ramon Vink ${ }^{\mathrm{b}}$, Luca Maresi ${ }^{\mathrm{b}}$ \\ a) TNO Science and Industrie, Stieltjesweg 1, 2628 CK Delft, the Netherlands; \\ b) ESA ESTEC, Noordwijk, the Netherlands
}

\begin{abstract}
Spectral features are introduced by the diffuser that is used during on-board sun calibration. New findings are presented on how to reduce the size of these spectral features. Reduction can be obtained via optical design of the calibration unit, but also in creating a better diffuser. A novel diffuser design will be presented and its performance will be compared to standard diffusers like an Aluminum diffuser, a Spectralon diffuser, and the QVD (Quasi Volume Diffuser). For the inhouse spectral features testing setup an improvement of about a factor of eight was obtained for the new diffuser type when compared to QVD. QVD in its turn is already better than an Aluminum diffuser by a factor of ten. Spectralon and QVD are found to be about equally good when measured in terms of spectral features reduction. The novel diffuser is referred to as SanDiff since it is a sandwich of QVD and PTFE material. For the SanDiff also the BSDF was measured and will be presented here.
\end{abstract}

The presented work was performed at TNO and was funded by ESA ESTEC.

Keywords: remote sensing, speckles, spectral features, diffuser, optical design, QVD, SanDiff

\section{INTRODUCTION}

Earth observation satellites operating in the UV, the visible, and IR spectral range measure the light from the sun, which is scattered by the earth and its atmosphere. The spectral contents of this scattered light yields information on the density of gases like Ozone $\left(\mathrm{O}_{3}\right)$ and Nitrite $\left(\mathrm{NO}_{2}\right)$. Since small changes in the contents of these gases are sought for the resolution with which the scattered intensity has to be measured is relatively high, i.e. in the order of $0.01 \%$ or better.

Modern satellites have one or more on-board diffusers for radiometric calibration purposes. The instrument can then be calibrated without observing the earth. The diffuser is illuminated directly by the sun and creates a homogeneous illumination at the entrance slit of the spectrometer. Division of measured spectra using once the diffuser, and once the earth, gives the absolute effect of absorption in the earth's atmosphere while division of two spectra obtained from the earth yield information on the changes in absorption in the atmosphere.

It has been recognized that Earth Observation missions that depend on Differential Optical AbSorption (DOAS) methods suffer from an undesired effect in the sun-normalised spectra, which is used in these methods. The effect causes large systematic errors (up to $100 \%$ ) in the retrieved slant columns of trace gases like $\mathrm{NO}_{2}$ and $\mathrm{BrO}$. Smaller errors are found for the larger absorbers.

For the GOME instrument it has been shown that the effect originates from the solar-irradiance spectra, which are used to normalise the earth-radiance spectra for DOAS applications. The effect has been studied in detail ${ }^{1}$ and it has been concluded that the on-board diffuser is responsible for the effect.

A similar effect has also been observed in (level 0) solar-irradiance spectra retrieved in-flight with the SCIAMACHY instrument ${ }^{2}$. The effect was discovered in 1998 during the on-ground calibration of the instrument, which took place at TNO. It is likely that the effect is present in all high-resolution spectrometers that utilise the classical type of on-board reflection diffusers for the acquisition of solar irradiance spectra. The observed variation in the solar irradiance spectra turns out not to be random but systematic and stable, with an annual cycle in amplitude and phase of the variations. The periodic pattern is specific for each of the atmospheric absorbers, i.e. for each fitting window.

This paper deals with the contributions to these fluctuations that are caused by the diffuser that scatters the light before it reaches the entrance slit of the spectrometer. The basic background of speckle theory will be presented in the first section. Averaging mechanisms to get rid of spectral features will be presented, followed by the presentation of the

Earth Observing Systems XIV, edited by James J. Butler, Xiaoxiong Xiong, Xingfa Gu

Proc. of SPIE Vol. 7452, 745203 - (c) 2009 SPIE · CCC code: 0277-786X/09/\$18 · doi: 10.1117/12.822957

Proc. of SPIE Vol. 7452 745203-1 
newly designed diffuser. In the next section the measurements and modelling on QVD and the new diffuser type will be shown. The match between simulation and measured data will be presented and discussed.

\subsection{Spectral Features}

What we refer to as Speckle Induced Spectral Features, or in short just Spectal Features, are those structures in the spectral data that originate from the speckles in the entrance slit of the spectrometer ${ }^{3}$. These fluctuations in intensity, specific to a certain wavelength band, are due to changes in the number of speckles in the entrance slit, and thus on the detector element. This number of speckles can change due to e.g. vibrations, changes in entrance angle of the light, wave front changes and aging of the diffuser.

\subsection{Spectral Features Amplitude}

Since the occurrence of Spectral Features is a statistical phenomenon the amplitude of the features as observed in the spectra obtained after division, can not be taken as its peak to peak value. The standard deviation of the spectral features spectrum is taken as a measure for the amount of spectral features present in that spectrum. The standard deviation of the spectral features spectrum will be referred to as Spectral Features Amplitude, i.e. the SFA.

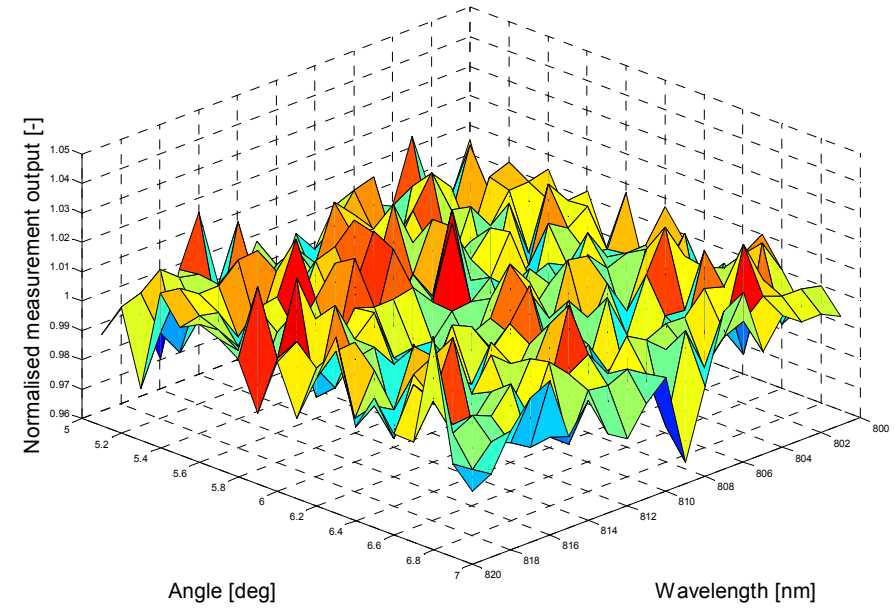

\section{Figure 1 Normalised detector output as a function of incidence angle and wavelength. The variation are the result of spectral features}

Figure 1 shows an impression of what a spectrum after normalization might look like. The intensity should ideally be unity. The wiggles are the Spectral Features and as can be seen they occur both as a function of wavelength and as a function of angle of incidence. The SFA can be calculated along both directions as given in Fig. 1.

\section{SPECKLE EFFECTS}

Speckles are usually only observed when lasers or other strongly coherent sources are used. Since the earth observation satellites are equipped with a high spectral resolution spectrometer, the intensity that is recorded per pixel on the camera has an effective coherence length that can be in the order of millimeters, and thereby large enough in order to have speckle effects. Since the coherence length depends also on the wavelength, the spectral features are more of an issue for longer wavelengths. 


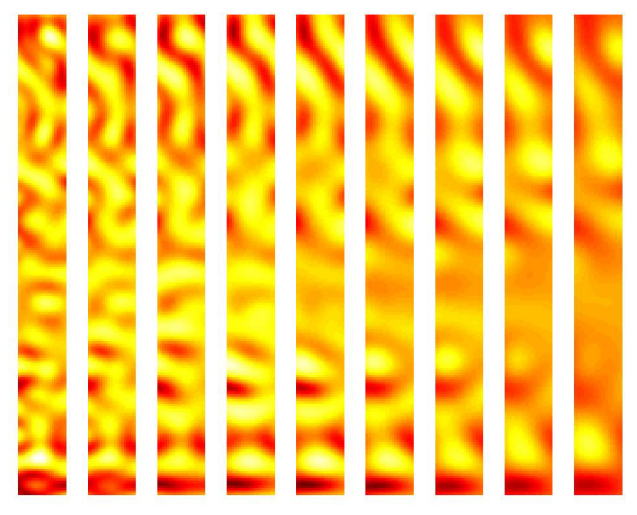

\section{Figure 2 Simulated speckle patterns in entrance slit of spectrometer. Wavelength changes from $400 \mathrm{~nm}$ on the left to $800 \mathrm{~nm}$ on the right hand side.}

The coherence length can be obtained via

$$
L_{c}=\frac{\lambda^{2}}{\Delta \lambda}
$$

where $\lambda$ stands for the wavelength used and $\Delta \lambda$ for the spectral bandwidth per detector pixel of the spectrometer.

The speckle diameter $d$ is given by

$$
d \propto \frac{\lambda}{N A},
$$

which indicates that speckles get larger for increasing wavelength and that the size decreases with increasing Numerical Aperture.

Spectral features occur due to the fact that the number of speckles per detector changes with, amongst other things, wavelength and illumination angle of the diffuser. The effect on the measured intensity will be larger in the case that there are only a few speckles than when there are many of them.

From these basic formulae it is clear that the number of speckles in the entrance slit is larger for shorter wavelengths. This means that the effect for one speckle more or less is stronger for longer wavelengths. This leads to the fact that spectral features will increase with increasing wavelengths. All averaging mechanisms found so far perform best for shorter wavelengths. This means that there is a certain wavelength above which no averaging takes place anymore and where the spectral features are thus purely defined by the number of speckles per detector pixel.

\section{SPECTRAL FEATURES REDUCING MECHANISMS}

In earth observation satellites that are in operation it has been observed that spectral features are an issue in the case that high accuracy absorption measurements are required. Several mechanisms to reduce the amplitude of the spectral features have been defined and will be discussed here.

The mechanisms can be divided into three categories:

1) optics design of the calibration unit

2) type of diffuser, special diffuser design

3) mode of operation 
Add 1) by optimizing the design of the calibration unit the spectral features amplitude can be greatly reduced. By increasing the numerical aperture the speckle size can be reduced resulting in a higher number of speckles in the entrance slit. This will lead to less effect when one speckle is removed from or added to the entrance slit opening. By using more length between the diffuser and the entrance slit the spectral features are also reduced. Especially a section where the light propagates from the diffuser to either a field plane or a pupil plane is very effective in reducing spectral features. These size effects are effective owing to the fact that the angle of incidence is not a constant and that the speckle distribution changes strongly with changing angle of incidence. The rate of change in speckle pattern with a change of angle of incidence increases with increasing lengths.

Add 2) The design of a diffuser can be optimized to make maximum use of angular effects and coherence effects. More on this topic in the section about the SanDiff design.

Add 3) The mode in which the diffuser is being used determines strongly the amount of features that are to be expected. Given a certain length between diffuser and spectrometer entrance slit several options are open to 'image' the diffuser onto the entrance slit. The first is to use no optics at all. In this case the feature will be very small but also the amount of light that reaches the entrance slit is very small. To get more light into the entrance slit the diffuser can be imaged onto the slit (Imaging mode) or the diffuser can be placed in the focal plane of a lens where the slit is in the other focal plane of the lens (FFT mode). Both imaging mode and FFT mode can be used but they show less effect for illuminating beam changes. The best option is to combine some length of free propagation with the FFT mode. By exchanging length between the free propagation mode and the FFT mode the angular averaging effect can be optimized. The imaging mode and FFT mode show hardly any wavelength dependence while free propagation shows a strong wavelength dependence. This wavelength dependency leads to strong inner pixel averaging, i.e. the period of the features as a function of wavelength in the spectrum is short when compared to the pixel bandwidth.

\section{SANDIFF DIFFUSER DESIGN}

To reduce the spectral features amplitude via special diffuser design will be the topic of the remainder of this paper. All numbers presented will only be valid for our in-house spectral features testing facility but the shown trends will in general be valid for all calibration unit designs.

To design a new type of diffuser several well known diffusers were taken as reference: simple surface diffuser like ground aluminum, volume diffuser like Spectralon, and the quasi volume diffuser QVD. These types of diffuser all have their own method to reduce the spectral features. A surface diffuser like aluminum produces the most spectral features since it has no means to reduce them. Spectralon and QVD show far smaller spectral features owing to the fact that they have built in reduction mechanisms. In the case of Spectralon it is the coherence length of the light that determines the number of independent speckle patterns that are created at the entrance slit. The coherence length is here defined by the wavelength and spectral bandwidth per pixel. More independent speckle patterns leads to a lower spectral features amplitude. This effect is referred to as the coherence effect. QVD has only two scattering interfaces where the first both reflects and transmits, and the second interface purely reflects. The relative large separation between these two scattering interfaces makes that angular changes results in a strong change in speckle pattern. The main spectral features reduction mechanism for QVD is therefore angular averaging. Owing to the opening angle of the sun of about 0.5 degrees QVD will already show strongly reduced spectral features. The spectral features will be reduced even further by the movement of the sun during the integration time of the detector.

The new diffuser, SanDiff, is a sandwich of two QVD's with a piece of PTFE material in between. The PTFE material is chosen to be thin enough to have a part of the incident light to pass through it into the second QVD. The first QVD is used mainly in transmission while the second one has a reflecting back surface. 


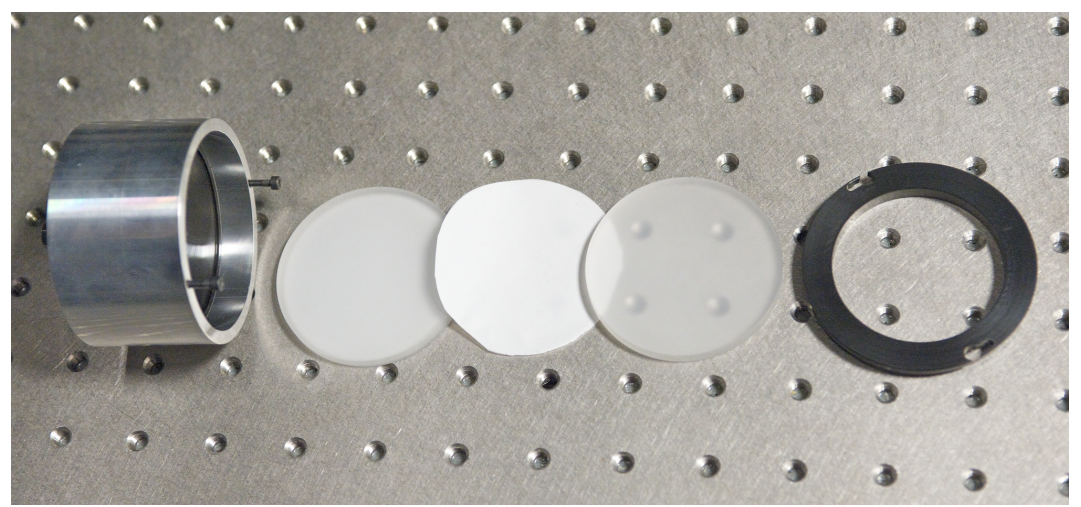

Figure 3 Prototype of the SanDiff diffuser. From left to right: holder, reflection QVD, PTFE sheet, transmission QVD, locking ring.

\section{SIMULATIONS AND MEASUREMENTS}

In order to predict what a new diffuser could do in terms of spectral features reduction a MatLab program was written to model the behavior of different diffusers in the optical configuration of our in-house setup. Apart from the layout of our in-house setup all other optical settings can be entered into the program such that it is a valuable tool for predicting the impact of calibration setups on spectral features effects.

Using the MatLab script several new diffuser designs were modeled and the SanDiff came out as one of the best options. Other designs that were about equal in performance were not selected for the testing campaign since they would require a lot of effort to get them space qualified. For the chosen SanDiff design the elements used are already space qualified so no issues in that respect are expected.

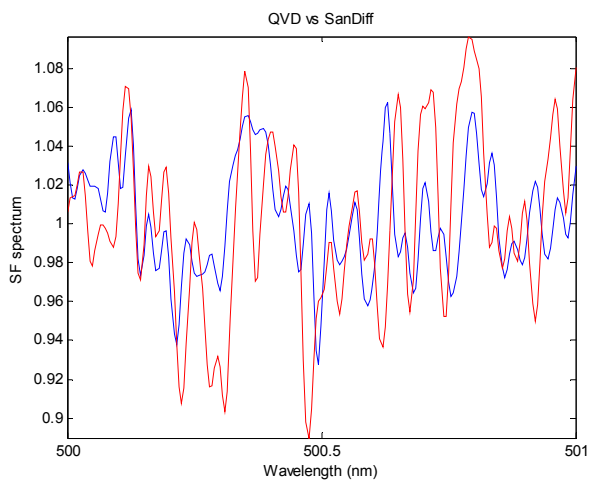

Figure 4 Spectral features spectrum as simulated for QVD (red) and SanDiff (blue)

\section{Table 1 Diffuser comparison simulation}

\begin{tabular}{|l|l|l|l|l|}
\hline Diffuser: & SFA & $\begin{array}{l}\text { Angular } \\
\text { sensitivity }\end{array}$ & $\begin{array}{l}\text { Wavelength } \\
\text { sensitivity }\end{array}$ & $\begin{array}{l}\text { Performance } \\
\text { relative to QVD } \\
\text { (lower= better) }\end{array}$ \\
\hline Aluminum & 6.03 & 0.09 & 1 & $\mathbf{1 2}$ \\
\hline QVD & 5.2 & 0.022 & 0.4 & $\mathbf{1}$ \\
\hline Double QVD & 4.29 & 0.015 & 0.275 & $\mathbf{0 . 4}$ \\
\hline SanDiff & 3.94 & 0.0097 & 0.135 & $\mathbf{0 . 1 2}$ \\
\hline
\end{tabular}


Table 1 gives the outcome of a comparison between different types of diffuser, e.g. aluminum, QVD, double QVD, and SanDiff. From these simulations the performances are compared to QVD. Aluminum performs a factor of 12 worse than QVD while SanDiff performs about a factor of 8 better than QVD.

The first column gives the static SFA values, i.e. no angular averaging nor inner pixel averaging. The second and third column give the sensitivities to angular and inner pixel averaging. The lower the figure, the better the diffuser will be. The fourth column is the product of the first three columns and normalized for a performance of 1 for QVD.

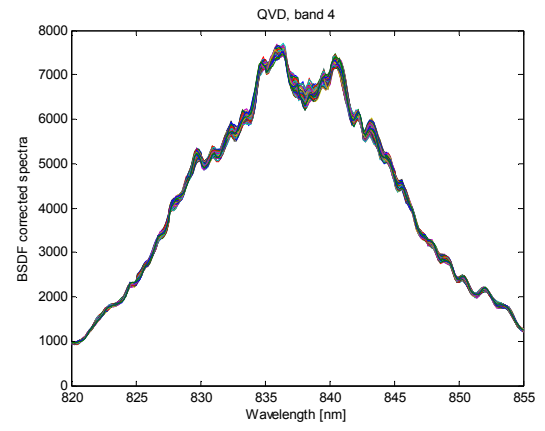

\section{Figure 5 Output spectrum from the SLD as used in the spectral features setup}

Experiments were performed in our in-house setup for diffuser characterization. The first results showed that the SanDiff is far better than QVD but the final level of performance could not be determined since SanDiff performed too good for the status of the setup. Our setup was designed to measure spectral features on aluminum, QVD and Spectralon. For those diffusers the setup was sufficiently stable. For the far smaller spectral features the requirements in terms of repetitions and integration time were too stringent such that the outcome was not good enough to get an absolute figure for the performance. The light sources as used in our setup were a QTH lamp and a Xenon lamp. The output intensities of these sources were relatively low, especially when taking into account that the dispersion yields a bandwidth per pixel of only $0.16 \mathrm{~nm}$. To overcome this measurement problem another type of light source was implemented into the system, an SLD (super luminescent diode). The emitting wavelength range is centered about $835 \mathrm{~nm}$ and has a bandwidth of about $40 \mathrm{~nm}$. This bandwidth is large enough to obtain full usage of the coherence effects of the SanDiff design.

The spectrum emitted by the SLD is shown in Fig.5. The different lines in that figure pertain to different illumination angles. Each spectrum is divided by the average of all those spectra, the result of which is shown in Fig.6. From the division the standard deviation is determined either in the wavelength direction for a given angle, or in the angular direction for a given wavelength. The obtained SFA values are independent from the analysis direction.
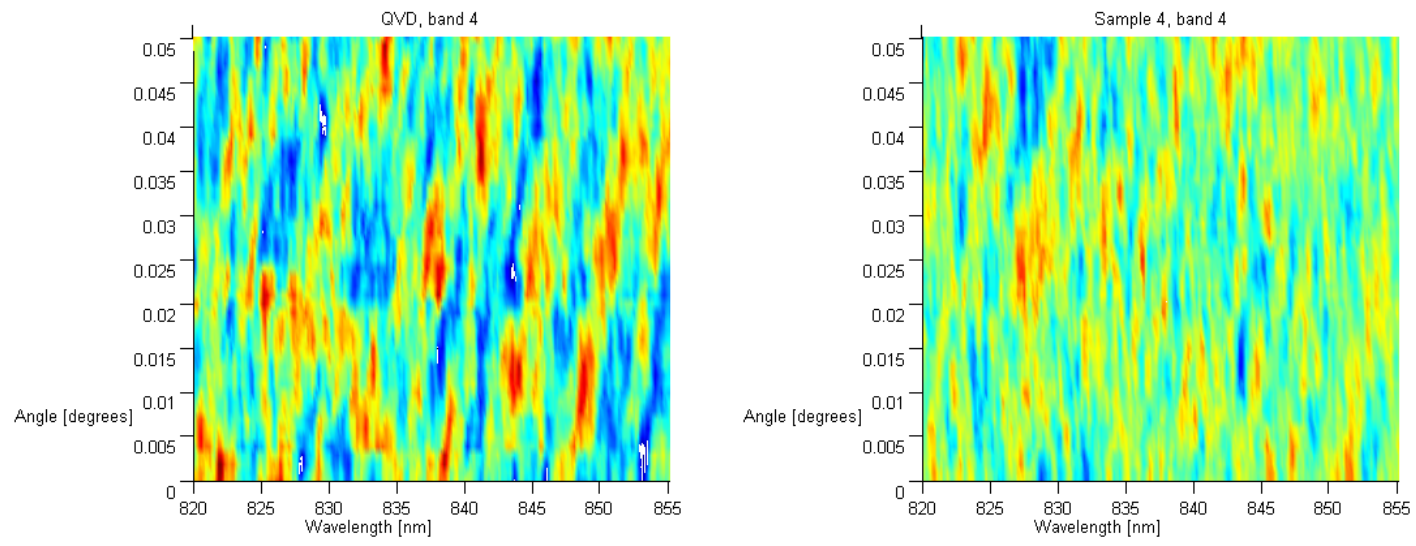

Figure 6 Spectra from QVD (Ihs) and SanDiff (rhs). Shown here as a function of angle and wavelength. The spectra are normalized by dividing them by the average of all spectra. 
Figure 6 shows the spectra after division by the spectrum obtained via averaging over all spectra. On the left hand side the SF spectrum is shown for the QVD (used as reference for this study), and at the right hand side the SF spectrum for the newly designed SanDiff. The average intensity is one and the scaling is identical for the left- and right-hand side figures. The darker colours for QVD indicate that the spectral features amplitude is larger for QVD than it is for SanDiff. The density of wiggles is higher for SanDiff meaning that angular averaging and inner pixel averaging will yield more reduction for SanDiff than it does for QVD.

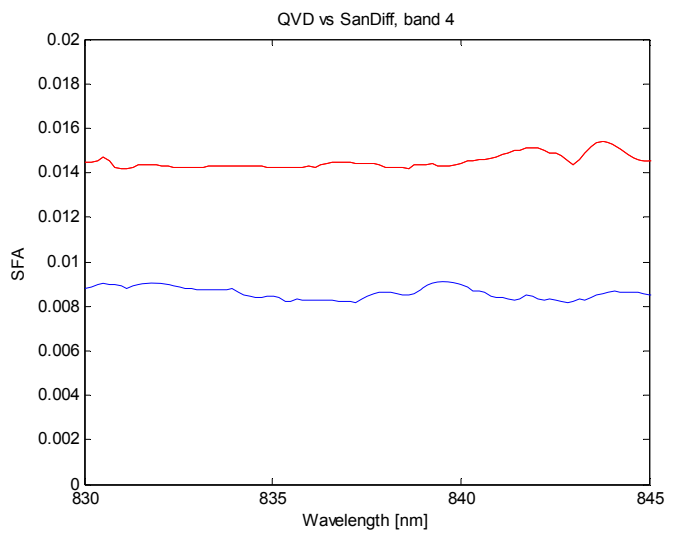

\section{Figure 7 SFA values for both QVD (red, top) and SanDiff (blue, bottom)}

In Fig.7 the SFA as a function of wavelength is shown for both QVD, the top curve, and for SanDiff, the bottom curve. The SFA calculation was performed here over a wavelength band of $20 \mathrm{~nm}$, and the resulting SFA value is plotted for the central wavelength. This calculation window is shifted over the full useful emission range of the SLD. As can be seen from Fig.7, the SFA for SanDiff is about a factor of 1.5 to 2 lower than that of QVD. During each measurement a static illumination direction was used and prior to a following measurement the angle of incidence was changed.

The output of the SLD is coupled into a single mode fibre, and the end facet of the fibre is placed in the front focal plane of an off-axis parabola. In this way a collimated beam is created that is used to illuminate the diffuser. Each area on the front surface of the diffuser is illuminated by a single angle. In real use of the diffuser the sun will be used as illuminating source. The opening angle of the sun of 0.5 degrees leads to a spread of illumination angles on the diffuser. This in its turn leads to angular averaging. Since the angular density of features is higher for SanDiff than it is for QVD (see Fig.6) by a factor of 3 to 4, the angular averaging for SanDiff will be stronger.

Combining the static ratio of SFA values between QVD and SanDiff (about 1.5 - 2) and the angular averaging effect (34), the overall gain in using SanDiff in stead of QVD will show a decrease in SFA of about a factor of $5-8$. The improvement of a factor of five is the minimum while at some wavelengths the feature density with respect to angle of incidence was so high that there an increase by a factor of eight is to be expected.

\section{DISCUSSION}

The improvement in SFA reducing capabilities of SanDiff when compared to QVD is very impressive. Before everybody thinks this is the answer to all spectral features issues one remark has to be made.

The improvement as reported here is only valid for our in-house setup. This means that if the real instrument has a similar optical arrangement and a similar spectral resolution and integration time, the improvement for that instrument when using SanDiff instead of QVD will also be about a factor of eight. All other optical arrangements will lead to a different improvement, possibly an even better improvement, but the change does not have to be positive.

The MatLab script we have developed allows us to make a prediction for other optical layouts and spectral resolutions. So far we have seen that the factor of eight is not yet the limit. For layouts where angular averaging is the main SFA reduction mechanism, the use of SanDiff will be very advantageous.

SanDiff stands for, as stated before, Sandwich Diffuser. This means that a SanDiff can not only be constructed using two QVD's and a thin piece of PTFE between it. Many other options have been formulated and tested in the model. The 
other options were not produced since the solutions were not readily space qualified where the presented SanDiff is space qualified by design.

Main criteria taken into account during the conceptual phase of the SanDiff design were: spectral range, incidence angle, diffusion angle, BSDF performance, SFA performance, physical size, ease of space qualification, volume/mass.

The shown SanDiff scores positively on all criteria. There is basically no limitation in the size in which it can be produced and the spectral range for which it can be used is equal to that of a standard QVD and Spectralon like diffusers. The BSDF measurements performed on the SanDiff show that the BSDF value is $0.18 / \mathrm{sr}$ (slightly higher than for a QVD $(0.15 / \mathrm{sr})$ but lower than that of Spectralon $(0.3 / \mathrm{sr})$.

The highest value is obtained in the specular direction but the BSDF drops only slightly as a function of angle, not more than $0.5 \%$ per degree.

The BSDF measurements show a good diffusing action, as had to be expected since the front element is a QVD. The mechanical holder limited the BSDF measurements due to a ring blocking the grazing incidence beams. The maximum angle that could be measured was about 40 degrees with the surface normal. Up to that angle the SanDiff was about Lambertian.

\section{CONCLUSIONS}

During the presented study new insights were obtained in the mechanisms to measure spectral features and in the way to model them. The new diffuser type, QVD/PTFE/QVD, referred to as SanDiff, has been measured extensively and from those measurements some short comings in our test facility became clear. A different light source, a super luminescent diode was required to measure the strongly reduced spectral features amplitude of this new type of diffuser. From these measurement the SFA reducing capability of the new diffuser was found to be better than that of QVD by a factor of 4.5 to 8 .

Based on the simulations and insight obtained in the physics behind spectral features generation and reduction, we expect that the SanDiff (QVD-PTFE-QVD) should yield a reduction in SFA of about a factor of eight.

The limited BSDF measurement performed on samples of the new type of diffuser show that their characteristics are virtually the same (the diffuser is reproducible) and that the scattering is to a high degree homogeneous (less than $0.5 \%$ intensity drop per degree) over the measured range. The BSDF of the SanDiff was higher than that of QVD. The obtained values were $0.18 / \mathrm{sr}$ and $0.15 / \mathrm{sr}$, respectively.

As conclusion it can be stated that the search for an improved QVD was successful. A new type of diffuser was obtained that shows a far better performance (up to eight times better) in terms of spectral features reduction as compared to diffusers that are presently being used. Its BSDF curves show that the diffuser acts as a proper diffuser in the sense that the output is homogeneous over the scattering angle, for all measured angles of incidence.

\section{REFERENCES}

[1] A. Richter, F. Wittrock, A. Ladstätter-Weißenmayer, J. Burrows "GOME measurements of stratospheric and tropospheric BrO", Advanced Space Research 29 (11), 1667-1672 (2002)

[2] B. Ahlers, G. Bazalgette Courrèges-Lacoste, C. Schrijvers, and H. van Brug, "In-orbit detection of Spectral Features in SCIAMACHY", In: Sensors, Systems, and Next-Generation Satellites VIII, Proceedings of the Meeting SPIE, 5570. Maspalomas, Spain, September 13-16, pp.401-410 (2004).

[3] H. van Brug, R. Vink, J. Groote Schaarsberg, G. Bazalgette Courrèges-Lacoste, and B. Snijders, "Speckles and their effects in spectrometers due to on-board diffusers", In : Earth Observing System IX, Proceedings of the Meeting SPIE, 5542. Denver, Colorado USA, August 2-6, pp. 334-341(2004) 